\title{
Oral intake of royal jelly improves anti-cancer effects and suppresses adverse events of molecular targeted therapy by regulating TNF- $\alpha$ and TGF- $\beta$ in renal cell carcinoma: A preliminary study based on a randomized double-blind clinical trial
}

\author{
YASUYOSHI MIYATA, KYOHEI ARAKI, KOJIRO OHBA, TOMHIRO MASTUO, \\ YUICHIRO NAKAMURA, TSUTOMU YUNO, YUTA MUKAI, ASATO OTSUBO, \\ KENSUKE MITSUNARI, YASUSHI MOCHIZUKI and HIDEKI SAKAI \\ Department of Urology, Nagasaki University Graduate School of Biomedical Sciences, \\ Nagasaki 852-8501, Japan
}

Received January 15, 2020; Accepted June 15, 2020

DOI: $10.3892 / \mathrm{mco} .2020 .2099$

\begin{abstract}
Molecular targeted therapies are commonly used in patients with metastatic renal cell carcinoma (RCC). However, the efficacy and safety of these therapeutic interventions require enhancement to improve prognosis in these patients. Royal jelly (RJ) has anti-cancer effects and adverse events across a variety of types of malignancy. The present study investigated the detailed mechanism underlying the effects of oral administration of RJ in patients with advanced RCC that were treated with molecular targeted agents in a randomized clinical trial. The study cohort comprised 16 patients treated with RJ and 17 patients treated with a placebo. Serum levels of tumor necrosis factor (TNF)- $\alpha$ and transforming growth factor (TGF)- $\beta$ were measured using enzyme-linked immunosorbent assays. The results of the present study demonstrated a larger decrease in tumor size upon supplementing patients with RJ following molecular targeted therapy compared with that in patients administered with the placebo. Patients exhibited reduced anorexia and fatigue in the RJ group compared with the placebo group. The relative dose intensity for patients in the RJ group was higher than that in patients in the placebo group. Post- and pre-treatment ratios of the serum levels of TNF- $\alpha$ and TGF- $\beta$ in patients in the RJ group were lower than those in patients in the placebo group, and these ratios correlated with decreasing tumor size and frequency of
\end{abstract}

Correspondence to: Dr Yasuyoshi Miyata, Department of Urology, Nagasaki University Graduate School of Biomedical Sciences, 1-7-1 Sakamoto, Nagasaki 852-8501, Japan

E-mail: yasu-myt@nagasaki-u.ac.jp

Key words: royal jelly, tumor necrosis factor- $\alpha$, transforming growth factor- $\beta$, molecular targeted agents, renal cell carcinoma anorexia or fatigue in patients. In conclusion, the results of the present study indicated that oral intake of RJ improved the efficacy and safety of molecular targeted therapy in patients with RCC and changed the levels of TNF- $\alpha$ and TGF- $\beta$ in the serum of patients, which is speculated to serve an important role in RJ-induced biological activities.

\section{Introduction}

Renal cell carcinoma ( $\mathrm{RCC}$ ) is a commonly occurring urological cancer that is characterized by a high metastatic potential at diagnosis and after radical surgery. Although various immunotherapies, including immune-checkpoint inhibitors, are used as standard therapy for patients with metastatic RCC, molecular targeted therapies, such as tyrosine kinase inhibitors (TKIs), are efficacious therapeutic interventions (1). However, the increase in overall survival of patients associated with these therapies are far from satisfactory. Therefore, understanding the functioning of new therapeutic agents is important and is a feat addressed by clinical trials $(2,3)$.

Royal jelly (RJ) is a milky secretion from the hypopharyngeal and mandibular glands of worker bees and possesses antimicrobial, anti-inflammatory, and antioxidant properties $(4,5)$. RJ is important in maintaining the quality of life and suppresses adverse events in patients undergoing anti-cancer therapies. RJ protects against organ dysfunctions and discomfort caused by various cancer therapies $(6,7)$. We have previously reported that RJ suppresses the adverse events caused by TKIs in patients with RCC (8). Several in vivo and in vitro studies have shown that $\mathrm{RJ}$ directly and indirectly exhibits anti-cancer effects in various malignancies (9-12). However, the detailed mechanisms employed by $\mathrm{RJ}$ in protecting against cancer and adverse events caused by anti-cancer therapy remains to be understand.

An important biological function of $\mathrm{RJ}$ is the regulation of inflammation and immunity $(4,5)$. Interestingly, inflammation 
and immunity are important for carcinogenesis and malignant invasiveness in multiple cancers $(13,14)$. Moreover, various pro-inflammatory cytokines, including tumor necrosis factor (TNF)- $\alpha$, tumor necrosis factor (TGF)- $\beta$, and interleukin (IL)-6 correlate with malignant transformation and occurrence of adverse events caused by anti-cancer therapies in various types of malignancies (15-22). Previous reports have shown that $\mathrm{RJ}$ regulates the synthesis of these pro-inflammatory cytokines (23-25). However, the correlation and mechanism employed by RJ in stimulating anti-cancer effects and suppressing adverse events by molecular targeted therapy in patients with RCC are yet to be elucidated.

We have previously shown that oral intake of RJ suppresses TKI-induced toxicity in patients with RCC in a randomized, double-blinded, placebo-controlled study (8). In this study, we investigated how orally administered RJ affects the anti-cancer effects induced by TKIs in the same patient cohort. Moreover, we analyzed the correlation between RJ-induced effects and changes in the serum levels of TNF- $\alpha$, TGF- $\beta$, and IL-6. Finally, we have demonstrated the benefits of administering RJ to advanced RCC patients awaiting TKI treatment in a preliminary clinical trial.

\section{Materials and methods}

Patients. Our study cohort consisted of 33 patients (23 males and 10 females) with RCC awaiting TKI treatment at the Nagasaki University Hospital (Nagasaki, China). The median (range) age at the time of treatment was 68 (54-79) years. There were 16 and 17 patients with a performance status of 0 and 1 , respectively. In our study population, 27 and 24 patients were diagnosed with high grade (Fuhrman grade 3 and 4) and high pT stage (pT3 and 4) cancer, respectively. All the patients had lymph node and/or distant metastasis. We used the clinicopathological features and eligibility criteria as per our previous report (8).

Study design. In this study, we performed a randomized, double-blind, placebo-controlled trial; patients were divided into two groups using computer-generated random numbers (17 in the placebo and 16 in the RJ group). Tumors were measured by computed tomography within the 3 months of the beginning and end of administering RJ or placebo. A group of patients was examined twice during the course of the study to check for adverse events. Tumor response was categorized based on the Response Evaluation Criteria in Solid Tumor version 1.1 as complete response (CR), partial response (PR), stable disease (SD), or progressive disease (PD) (26). Toxicity was evaluated using the Common Terminology Criteria for Adverse Events version 5.0 by the National Cancer Institute. In this study, adverse events were divided into two groups (absence or presence of and Grade 1-4) regardless of severity owing to the relatively small cohort. Serum levels of TNF- $\alpha$, TGF- $\beta$, and IL- 6 were quantified by enzyme-linked immunosorbent assay (R\&D systems, Inc.; MN) before and after 3 months of treatment.

Protocol. As shown in our previous report (8), the starting dose of sunitinib, pazopanib, axitinib, and sunitinib was 50, 800, 10 , and $800 \mathrm{mg} /$ day, respectively. Upon observing intolerable adverse events, the doses were decreased to 25.0-37.5,
400-600, 5, and $400 \mathrm{mg}$ /day, respectively. TKI administration was stopped once continuous intolerable adverse events were observed. Other molecular targeted therapies, including TKIs and/or m-TOR inhibitors, were administered as soon as possible in all the patients. Patients with a rest period of over 30 days were excluded from this study. We did not use immune check-point inhibitors owing to the lack of approval for treatment of RCC at the time. Relative dose intensity (RDI) was calculated as the ratio of 'delivered' to 'planned' dose intensity. In short, $100 \%$ RDI means that the TKI was administered at the dose mentioned in the original protocol.

RJ was procured from Yamada Agriculture Center Inc (Okayama, Japan). RJ and placebo were prepared as capsules containing $900 \mathrm{mg}$ RJ and starch, respectively. They were similar in taste, smell, size, shape, and color. Capsules were administered orally four times per day (after breakfast, lunch, and dinner and before bedtime) for three months.

The study protocol was approved by the Human Ethics Review Committee of Nagasaki University Hospital (Nagasaki, Japan; No. 15102604-2 and it was registered as UMIN000020152). All experiments complied with the principles embodied in the Declaration of Helsinki. All the patients provided written informed consent to participate in all aspects of the study. This was a double-blind study.

Statistical analysis. Results have been expressed as the mean and standard deviation for data with normal distribution or median and interquartile range for data with non-normal distribution. Student's t-test and Mann-Whitney U test was used to compare the continuous variables in data with normal and non-normal distribution, respectively. Multiple comparisons of the data were analyzed by Scheffé's method. ANOVA was performed prior to Scheffe's method. Chi-square test was used for categorical comparison of the data. All the statistical analyses were two-sided, significance was set at $\mathrm{P}<0.05$, and were performed on a personal computer using the statistical package StatView for Windows (version 5.0, Abacus Concept, Inc., Berkeley, CA).

\section{Results}

Patient background. As shown in our previous report (8), $21,7,4$, and 1 patient(s) were treated with sunitinib, pazopanib, axitinib, and sorafenib, respectively: There was no significant difference between the patients in the placebo and RJ-administered groups $(\mathrm{P}=0.539)$. Moreover, the patients showed similar clinicopathological features including age $(\mathrm{P}=0.101)$, sex $(\mathrm{P}=0.909)$, performance status $(\mathrm{P}=0.598)$, grade of cancer $(\mathrm{P}=0.425)$, pT stage $(\mathrm{P}=0.201)$, and metastasis to the lymph node $(\mathrm{P}=0.881)$ and distant organs $(\mathrm{P}=0.325)$.

Tumor response and adverse events. No patient exhibited CR within 2-3 months of administering the TKI or placebo. One patient exhibited CR after intake of RJ. The frequency of patients with PR in the RJ group (50.0\%) was twice as much as that in the placebo group (17.6\%). Compared to placebo-administered patients, no patient exhibited PD in the RJ group. Finally, our data demonstrated that oral intake of RJ improved the anti-cancer effects 3 months after TKI treatment ( $\mathrm{P}=0.037$; Table I). 
Table I. Three month response.

\begin{tabular}{lccc}
\hline Response & Total & Placebo & Royal jelly \\
& & & P-value \\
Complete response; N (\%) & $1(3.0)$ & $0(0.0)$ & $1(6.3)$ \\
Partial response & $11(33.3)$ & $3(17.6)$ & $8(50.0)$ \\
Stable disease & $16(48.5)$ & $9(52.9)$ & $7(43.8)$ \\
Progressive disease & $5(15.2)$ & $5(29.4)$ & $0(0.0)$ \\
\hline
\end{tabular}

Table II. Frequency of anorexia and fatigue.

\begin{tabular}{lrrr}
\hline Factor & Placebo & Royal jelly & P-value \\
\hline Anorexia & & & 0.009 \\
Absence & $4(23.5)$ & $11(68.8)$ & \\
Presence & $13(76.5)$ & $5(31.2)$ & \\
Fatigue & & & $<0.001$ \\
Absence & $2(11.8)$ & $11(68.8)$ & \\
Presence & $15(88.2)$ & $5(31.2)$ & \\
\hline
\end{tabular}

Hypertension was among the most common adverse events $(n=23 ; 67.9 \%)$; fatigue $(n=20 ; 60.6 \%)$, anorexia $(n=18 ; 60.0 \%)$, and hand-foot syndrome $(\mathrm{n}=18 ; 60.0 \%)$ were the other common adverse events. Blood tests revealed leukopenia, anemia, renal dysfunction, liver dysfunction, and thyroid abnormality in $11(36.7 \%), 9$ (27.3\%), 15 (45.5\%), 7 (21.2\%), and 14 patients (42.4\%), respectively. Among these adverse events, anorexia and fatigue in patients in the RJ group was significantly lower compared to that in the placebo group $(\mathrm{P}=0.009$ and $\mathrm{P}<0.001$, respectively; Table II). Detailed information on the adverse events caused by TKI treatment have been described previously (8).

Relative dose density. As shown in Fig. 1, 1-month RDI (mean/standard deviation; 88.6/21.2\%) in the patients in the RJ group was significantly higher $(\mathrm{P}=0.007)$ than that in patients administered placebo (68.6/28.7\%). After 2 months of treatment, TKI treatment was discontinued and decreased in $15(88.2 \%)$ and 8 patients $(50.0 \%)$ in the RJ and placebo groups, respectively; 2-month RDIs for the patients in the RJ and placebo groups showed similar differences (71.9/43.2 and $40.4 / 40.1 \%$, respectively; $\mathrm{P}=0.031$; Fig. 1 ). At the end of the study period (3 months), the initial dose administered was maintained in $7(43.8 \%)$ and 2 patients $(11.8 \%)$ in the RJ and placebo groups, respectively. The 3-month RDI in patients in the RJ group was slightly higher than that in the placebo group; however, this difference was not significant $(\mathrm{P}=0.114$ and 0.084 , respectively; Fig. 1).

Correlation between serum levels of TNF- $\alpha, T G F-\beta$, and IL-6 and tumor response. Prior to treatment with TKI, serum levels of TNF- $\alpha$ in patients in the RJ group [mean/standard deviation and median (interquartile range) were 5.90/5.46 and 5.02 (0.00-11.60)] was higher than that in placebo-administered patients [2.37/3.49 and $2.37(1.17-5.83)]$. However, this

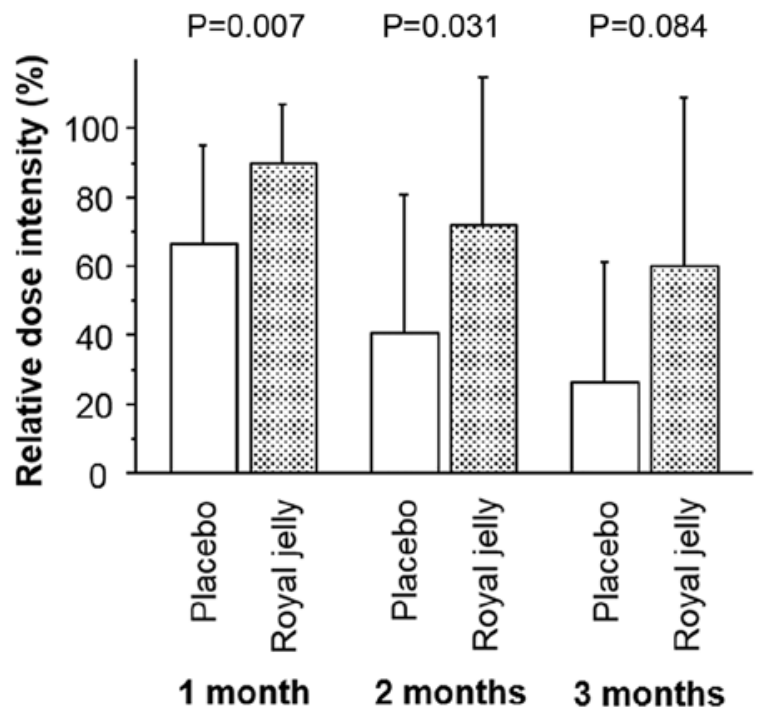

Figure 1. Relative dosage of the tyrosine kinase inhibitors after 1, 2, and 3 months of starting the treatment.

difference was not significant $(\mathrm{P}=0.378)$. Similarly, patients in the RJ and placebo groups showed no significant difference in the serum levels of IL-6 [10.69/13.21 and 4.52 (1.62-18.20) versus $9.57 / 13.28$ and $2.13(1.55-11.97), \mathrm{P}=0.564]$ and TGF- $\beta$ [537.14/226.18 and 532.00(324.41-707.83) versus 600.77/309.34 and 543.78 (360.38-786.93), $\mathrm{P}=0.719]$.

Post- and pre-treatment ratios of these variables in patients in the RJ and placebo groups are shown in Fig. 2. The ratio of the levels of TNF- $\alpha$ in patients of the RJ group was significantly lower than that in patients in the placebo group $(\mathrm{P}=0.007$; Fig. 2A). The serum levels of TGF- $\beta$ showed a similar trend $(\mathrm{P}=0.006$; Fig. 2B). However, there was no significant difference between the decreased serum levels of IL- 6 in the RJ and placebo groups ( $\mathrm{P}=0.221$; Fig. $2 \mathrm{C})$. Next, we determined the correlation between the decreased variables and tumor response upon TKI treatment by Scheffé's method (Fig. 2D-2F). Post- and pre-treatment ratios of the levels of TNF- $\alpha$ in patients exhibiting PR was significantly lower than that in patients exhibiting SD and $\mathrm{PD}(\mathrm{P}=0.018$ and 0.002 , respectively; Fig. 2D). Similar trends, albeit non-significant, were observed for the levels of TGF- $\beta$ between patients exhibiting PR and SD or PD $(\mathrm{P}=0.333$ and 0.219 , respectively; Fig. 2E). The decrease in serum IL-6 did not correlate with tumor response (Fig. 2F).

Correlation between serum levels of TNF- $\alpha, T G F-\beta$, and IL-6 and adverse events. Post- and pre-treatment ratios of the serum 

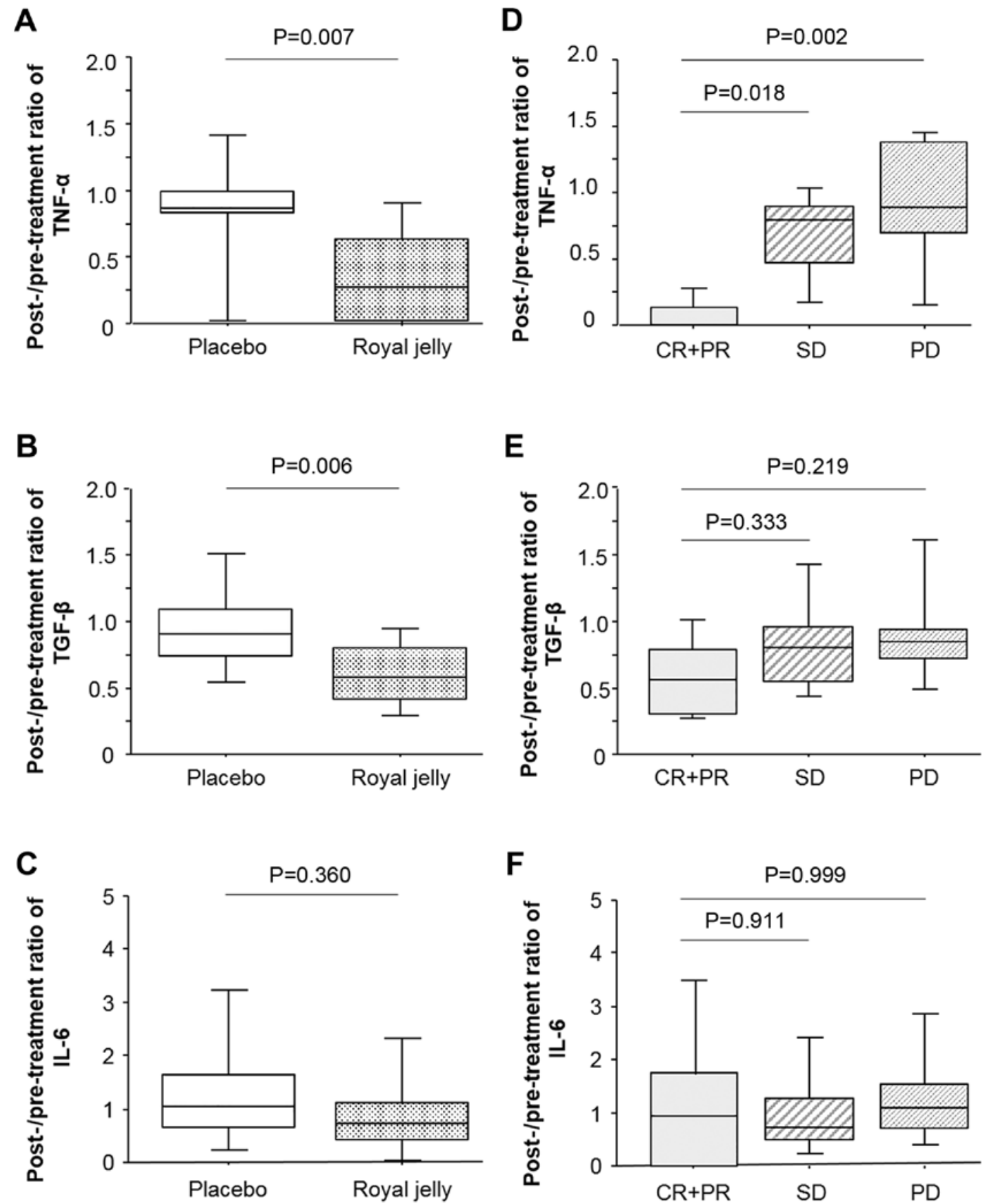

Figure 2. Post/pre-treatment ratio of the levels of (A) TNF- $\alpha$, (B) TGF- $\beta$ and (C) IL-6 in the serum of patients in the placebo and royal jelly groups. Correlation between the ratios and tumor response after molecular targeted therapy and the ratios of (D) TNF- $\alpha$, (E) TGF- $\beta$ and (F) IL-6. Lower and upper whiskers indicate the minimum and maximum, respectively, of the data set. The box is drawn from the lower quartile to the upper quartile with a horizontal line drawn in the middle to denote the median. TNF, tumor necrosis factor; TGF, transforming growth factor; IL, interleukin.

levels of TNF- $\alpha$ did not correlate with anorexia (Fig. 3A). The serum levels of TGF- $\beta$ in patients with anorexia was significantly higher $(\mathrm{P}=0.030)$ as compared to those without anorexia (Fig. 3B). Similar to TNF- $\alpha$, there was no significant correlation between the post- and pre-treatment ratios of the serum levels of IL-6 and anorexia (Fig. 3C). Similar results were observed for patients with fatigue (Fig. 3D-F): The post- and pre-treatment ratio of the serum levels of TGF- $\beta$ correlated with fatigue $(\mathrm{P}=0.030)$ whereas that of TNF- $\alpha$ and IL-6 did not.

There was no significant difference between the post- and pre-treatment ratios of the serum levels of TGF- $\beta$, TNF- $\alpha$, and IL- 6 and other adverse events like digestive symptoms $(\mathrm{P}=0.719,0.743$, and 0.439 , respectively), hypertension ( $\mathrm{P}=0.389,0.089$, and 0.938 , respectively), and oral mucositis ( $\mathrm{P}=0.338,0.346$, and 0.985 , respectively). Finally, based on our results, a schematic summarizing the main results is shown in Fig. 4.

\section{Discussion}

This study demonstrated that oral administration of RJ improves tumor response in patients with advanced RCC undergoing TKI treatment till 3 months of beginning the 
A

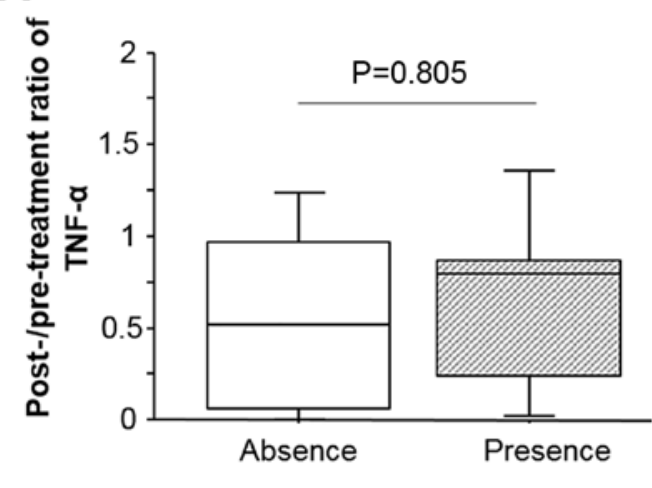

Anorexia

B

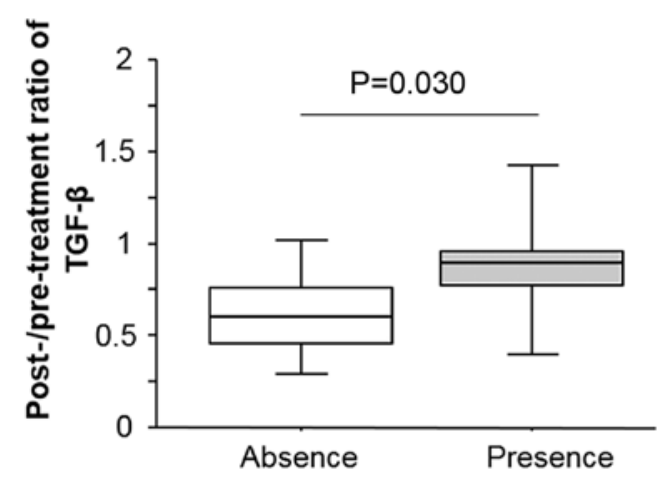

C

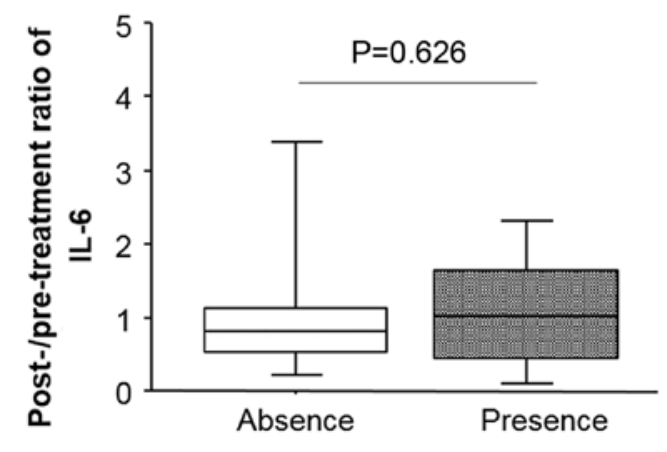

Anorexia
D

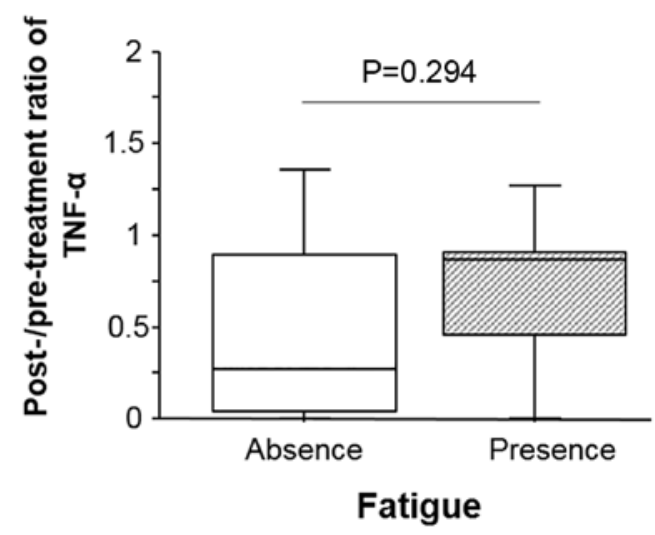

E
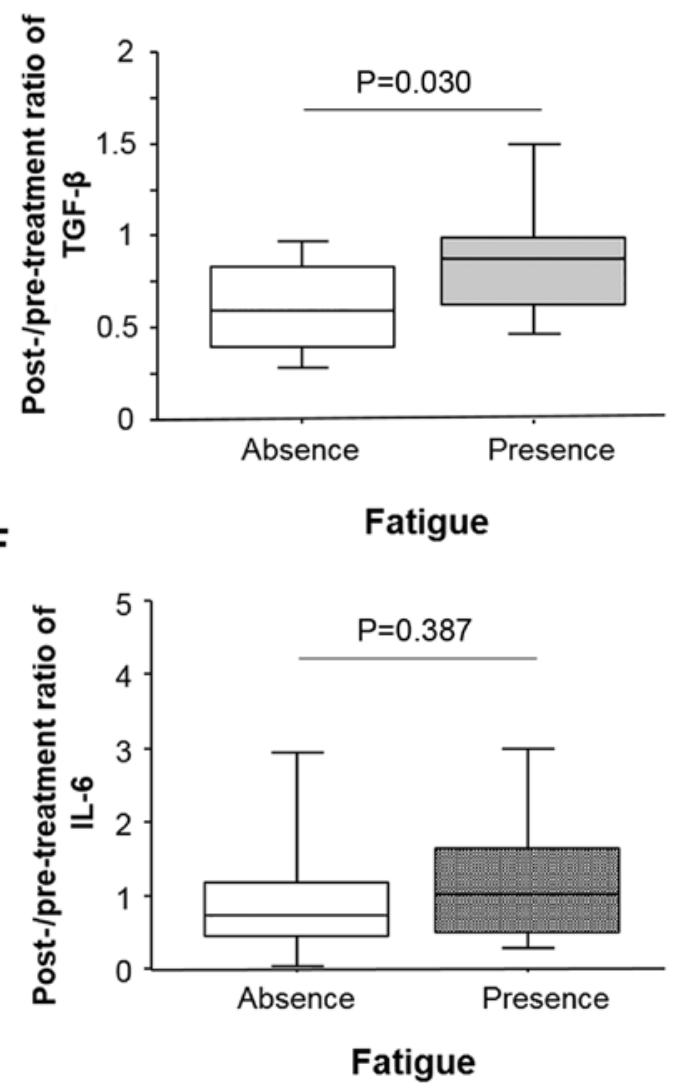

Figure 3. Correlation between the post- and pre-treatment ratios of the serum levels of (A) TNF- $\alpha$, (B) TGF- $\beta$, and (C) IL-6 with anorexia. The correlations of the serum levels of (D) TNF- $\alpha$, (E) TGF- $\beta$, and (F) IL- 6 with fatigue. Lower and upper whiskers indicate the minimum and maximum, respectively, of the data set. The box is drawn from the lower quartile to the upper quartile with a horizontal line drawn in the middle to denote the median. TNF, tumor necrosis factor; TGF, transforming growth factor; IL, interleukin.

molecular targeted therapy. To the best of our knowledge, this is the first study on the anti-cancer effects of RJ in patients with RCC. Several in vivo and in vitro studies have reported the anti-cancer effects of RJ in malignancies $(9,11)$. $\mathrm{RJ}$ reduces proliferation of the MCF-7 breast cancer cells, especially in estrogen-induced proliferating MCF-7 cells (9). The major fatty acid 10-hydroxy-2-decenoic acid (10-HAD) inhibits the WiDr colon cancer cells upon treatment with RJ in a dose-dependent manner (12); the lipophilic fraction of RJ possesses anti-proliferative activity against the SH-SY5Y human neuroblastoma cells (10). Oral administration of RJ inhibits the growth of the 4T1 mouse mammary tumor cells in a mouse model of breast cancer (11). Thus, we speculated that RJ directly affects proliferation of RCC cells.

Several reports have shown the indirect mechanisms involved in the anti-cancer effects of RJ, such as changes in tumor microenvironments and immune response in various cancers $(11,12,27)$. Our results showed that oral intake of RJ decreased the serum levels of TNF- $\alpha$; their levels in the serum in patients exhibiting CR and PR was remarkably lower than those in patients exhibiting SD or PD. In vitro studies have shown that RJ reduces TNF- $\alpha$ synthesis from macrophage cells $(24,28)$. RJ inhibits TNF- $\alpha$ production in mouse peritoneal macrophages stimulated by lipopolysaccharide and 


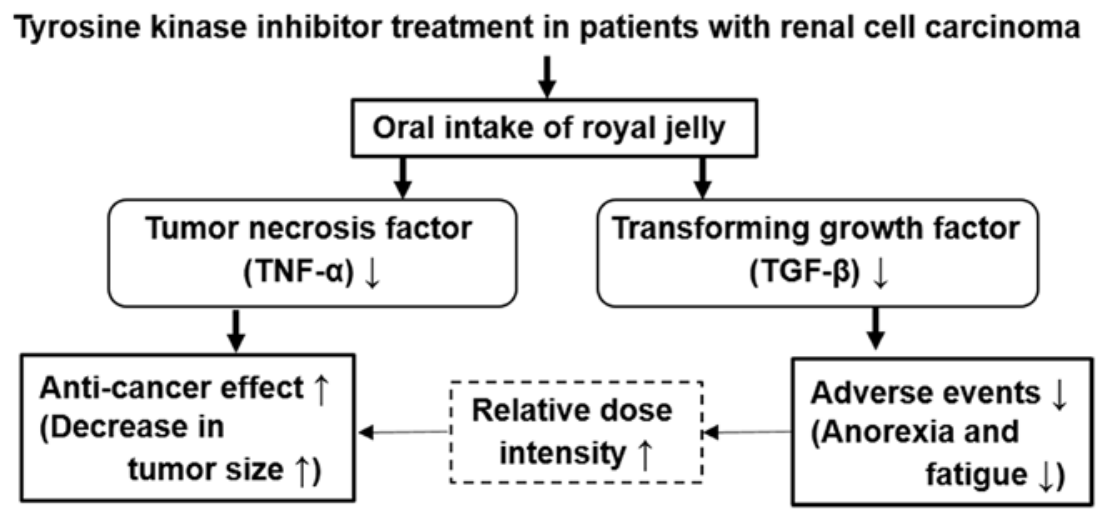

Figure 4. Scheme of the increase in anti-cancer effects and suppression of adverse events after royal jelly intake via regulation of TNF- $\alpha$ and TGF- $\beta$ in tyrosine kinase inhibitor treatment in renal cell carcinoma. TNF, tumor necrosis factor; TGF, transforming growth factor.

interferon- $\gamma$ (23). Combining 10-HAD with RJ suppresses the production of TNF- $\alpha$ in colon cancer cells (12). Serum levels of TNF- $\alpha$ in rats treated with cyclophosphamide and RJ was significantly lower than those with only cyclophosphamide treatment (29). Thus, our results of the decreased serum levels of TNF- $\alpha$ upon RJ treatment was in accordance with that of previous studies.

TNF- $\alpha$ is a major mediator of cancer-induced inflammation in the tumor microenvironment and malignant transformation by regulating various types of biomolecules and chemokines (15). Several in vivo and in vitro studies have demonstrated that TNF- $\alpha$ levels correlate with risk of cancer, tumor growth, invasion, and metastasis of RCC $(18,21,30)$. Furthermore, various anti-cancer agents suppress the malignant transformation and tumor progression by interfering with TNF- $\alpha$-related functions in malignancies including $\operatorname{RCC}(22,31,32)$. A clinical trial demonstrated that intravenously injecting infliximab, an anti-TNF- $\alpha$ monoclonal antibody, decreases tumor size and prognosis in RCC patients, suggesting that TNF- $\alpha$ is a putative therapeutic target in such patients (33). Thus, decreasing $\mathrm{TNF}-\alpha$ levels suppresses malignant invasiveness and enhances anti-cancer effects in RCC among other cancers. TNF- $\alpha$ is pivotal in the resistance against sunitinib treatment in RCC (18). A high expression of CD44 correlated with worse outcomes of sunitinib treatment in 25 patients with metastatic RCC with TNF- $\alpha$ as one of the key modulators; CD44 expression induced by TNF- $\alpha$ contributes to acquiring resistance to sunitinib treatment (18). Although our study population was treated with various TKIs, we speculate that decreasing TNF- $\alpha$ may be important in eliciting anti-cancer effects in RCC patients. Also, TNF- $\alpha$-related anti-cancer effects could have been induced by oral intake of $\mathrm{RJ}$ in our cohort. Unfortunately, there is no scope to discuss and conclude this hypothesis since there is no known correlation between RJ, TNF- $\alpha$, and anti-cancer effects in patients with RCC. A study recently showed that the synthesis of TNF- $\alpha$ is inhibited by 10-HAD in human colon cancer cells (12). More recently, it has been shown that purified proteins from RJ improved the damage caused to necrotic hepatocytes by reducing the levels of TNF- $\alpha$; this phenomenon correlated with anti-cancer effects in the HepG2 hepatocellular cancer cells (29).

RDI may have regulated the anti-cancer effects in our study population. We observed that a percentage of patients treated with the initial dose versus patients treated with RJ was significantly higher as compared to those administered placebo. The 1-month and 2-month RDIs for patients in the RJ group were significantly higher than those in the placebo groups. The dose intensity correlated with anti-cancer effects induced by multiple types of anti-cancer agents used for malignancies. Continuing sunitinib therapy for more than one course and maintaining more than $60 \%$ of 1-month RDI correlated with better outcome for progression-free survival in patients with metastatic RCC (34). Similarly, more than $65 \%$ of two course-RDI should be maintained for optimal therapeutic efficacy of sunitinib treatment (35). Taken together, higher intensities of TKIs upon oral administration of RJ dictates better anti-cancer effects in patients with RCC. We also speculated that suppression of TKIs induced toxicities by RJ is important in maintaining the RDI and stimulated anti-cancer effects in our study cohort. Several reports have shown that successfully managing drug-induced toxicities leads to the long-term continuation of sunitinib and positively correlates with anti-cancer effects in various types of cancers including RCC $(36,37)$. We have previously demonstrated that oral intake of RJ suppresses the frequency and severity of several adverse events, such as anorexia and fatigue, in RCC patients treated with TKIs (8); and this study confirmed in this study used by different criterion. However, the detailed mechanism employed by mediating these protective effects remains to be studied.

To understand the protective effects mediated by RJ against adverse events in TKI-treated patients, we focused on the levels of TNF- $\alpha$, TGF- $\beta$, and IL- 6 in patients with advanced RCC. We found that significant decrease in the serum levels of TGF- $\beta$ level upon oral intake of RJ significantly correlated with the suppression of adverse events, such as fatigue and anorexia. TGF- $\beta$ promotes chronic fatigue syndrome and cancer-induced cachexia including anorexia $(16,20,38)$. The serum/plasma levels of TGF- $\beta$ increases in patients with chronic fatigue syndrome and/or cancer-induced anorexia $(16,19,20)$. Furthermore, in a murine model of pancreatic cancer cachexia, pharmacologic blockage of TGF- $\beta$ improves cancer-related cachexia including weight loss and skeletal muscle proteolysis (17). Inhibiting TGF- $\beta$ did not affect cancer cell proliferation and subcutaneous tumor growth (17). Although there is no clear correlation between 
TGF- $\beta$ and $\mathrm{RJ}$ in vivo, oral intake of RJ reverses TGF- $\beta$ levels in the bronchoalveolar lavage fluid induced by bleomycin treatment in rats (25). These findings are in accordance with our results that RJ-induced decrease in TGF- $\beta$ levels correlates with improved anorexia and fatigue but does not correlate with tumor response after TKI treatment in RCC patients. RJ and 10-HDA inhibit IL-6 production in macrophages $(23,24)$; however, there is little information on the influence of RJ on the serum levels of IL- 6 in cancer patients. Our results showed that oral administration of RJ did not affect serum IL-6 levels in patients with RCC. However, this is preliminary and more detailed studies are necessary to conclude this.

Although this is the first clinical trial on the oral administration of RJ in RCC patients and its effects on anti-cancer activities and adverse events, this study has several limitations. The most important is the small cohort of patients in the placebo and RJ groups. Another limitation is the use of multiple TKIs and patients with non-uniform characteristics. Moreover, the RJ capsules were provided by a company that sell supplements made from honey including RJ. Therefore, to avoid any bias, we conducted this trial using a double-blind randomized analysis; selection of administration, data collection, and analysis were performed by a third party approved by our IRB. Furthermore, RJ was administrated only for 3 months. We did not observe any adverse events induced by RJ in our study cohort. Therefore, we emphasize the need for detailed clinical trials with longer period of treatment in the future to evaluate the treatment strategies in patients with advanced RCC.

In conclusion, oral administration of $\mathrm{RJ}$ strengthened anti-cancer effects of molecular targeted agents in patients with metastatic RCC. Moreover, it suppressed the frequency of anorexia and fatigue induced by TKI therapy. RJ was useful in maintaining the RDI compared to patients with placebo. Furthermore, RJ decreased the serum levels of TNF- $\alpha$ and TGF- $\beta$ and such changes were speculated to correlate with the enhanced tumor response and suppressed adverse events.

\section{Acknowledgements}

The authors would like to thank Ms. Mitsuko Yoneda (Nagasaki University Hospital) for providing technical support.

\section{Funding}

This study was supported by a Yamada Research grant (grant no. 2015-20; to YMi).

\section{Availability of data and materials}

The datasets used and/or analyzed during the present study are available from the author on reasonable request.

\section{Authors' contributions}

YMi conceived and designed the experiments, performed data analysis, and contributed to the writing of the manuscript. KA performed the experiments and contributed to sample collection and writing of the manuscript. KA, YN, TY, YMu and $\mathrm{AO}$ performed the experiments and analyzed the data.
$\mathrm{KM}, \mathrm{TM}, \mathrm{KO}$ and YMo contributed to sample and clinical data collection. HS conceived and designed the experiments and contributed to drafting and revising the manuscript. All authors read and approved the final manuscript.

\section{Ethics approval and consent to participate}

The purpose of the present study was explained to the participants, who all signed written consent forms prior to participating in the study. This study design was approved by the Institutional Review Board of Nagasaki University Hospital (Nagasaki, Japan).

\section{Patient consent for publication}

Not applicable.

\section{Competing interests}

The authors declare that they have no competing interests.

\section{References}

1. Zarrabi $\mathrm{K}$ and Wu S: Current and emerging therapeutic targets for metastatic renal cell carcinoma. Curr Oncol Rep 20: 41, 2018.

2. Chang AJ, Zhao L, Zhu Z, Boulanger K, Xiao H, Wakefield MR, Bai Q and Fang Y: The past, present and future of immunotherapy for metastatic renal cell carcinoma. Anticancer Res 39: 2683-2687, 2019.

3. Zhu N, Weng S, Wang J, Chen J, Yu L, Fang X and Yuan Y: Preclinical rationale and clinical efficacy of antiangiogenic therapy and immune checkpoint blockade combination therapy in urogenital tumors. J Cancer Res Clin Oncol 145: 3021-3036, 2019.

4. Almeer RS, Soliman D, Kassab RB, AlBasher GI, Alarifi S, Alkahtani S, Ali D, Metwally D and Abdel Moneim AE: Royal jelly abrogates cadmium-induced oxidative challenge in mouse testes: Involvement of the Nrf2 pathway. Int J Mol Sci 19: 3979, 2018.

5. Petelin A, Kenig S, Kopinč R, Deželak M, Černelič Bizjak M and Jenko Pražnikar Z: Effects of royal jelly administration on lipid profile, satiety, inflammation, and antioxidant capacity in asymptomatic overweight adults. Evid Based Complement Alternat Med 2019: 4969720, 2019.

6. Miyata Y and Sakai H: Anti-Cancer and protective effects of royal jelly for therapy-induced toxicities in malignancies. Int $\mathrm{J}$ Mol Sci 19: 3270, 2018.

7. Münstedt $\mathrm{K}$ and Männle $\mathrm{H}$ : Using bee products for the prevention and treatment of oral mucositis induced by cancer treatment. Molecules 24: 3023, 2019.

8. Araki K, Miyata Y, Ohba K,Nakamura Y, Matsuo T, Mochizuki Y and Sakai H: Oral intake of royal jelly has protective effects against tyrosine kinase inhibitor-induced toxicity in patients with renal cell carcinoma: A randomized, double-blinded, placebo-controlled trial. Medicines (Basel) 6: 2, 2018.

9. Nakaya M, Onda H, Sasaki K, Yukiyoshi A, Tachibana H and Yamada K: Effect of royal jelly on bisphenol A-induced proliferation of human breast cancer cells. Biosci Biotechnol Biochem 71: 253-255, 2007.

10. Gismondi A, Trionfera E, Canuti L, Di Marco G and Canini A: Royal jelly lipophilic fraction induces antiproliferative effects on SH-SY5Y human neuroblastoma cells. Oncol Rep 38: 1833-1844, 2017.

11. Zhang S, Shao Q, Geng $\mathrm{H}$ and Su S: The effect of royal jelly on the growth of breast cancer in mice. Oncol Lett 14: 7615-7621, 2017.

12. Yang YC, Chou WM, Widowati DA, Lin IP and Peng CC: 10-hydroxy-2-decenoic acid of royal jelly exhibits bactericide and anti-inflammatory activity in human colon cancer cells. BMC Complement Altern Med 18: 202, 2018.

13. Mantovani A, Allavena P, Sica A and Balkwill F: Cancer-related inflammation. Nature 454: 436-444, 2008. 
14. Jiang $X$ and Shapiro DJ: The immune system and inflammation in breast cancer. Mol Cell Endocrinol 382: 673-682, 2014.

15. Sethi G, Sung B and Aggarwal BB: TNF: A master switch for inflammation to cancer. Front Biosci 13: 5094-5107, 2008.

16. Blundell S, Ray KK, Buckland M and White PD: Chronic fatigue syndrome and circulating cytokines: A systematic review. Brain Behav Immun 50: 186-195, 2015.

17. Greco SH, Tomkötter L, Vahle AK, Rokosh R, Avanzi A, Mahmood SK, Deutsch M, Alothman S, Alqunaibit D, Ochi A, et al: TGF- $\beta$ blockade reduces mortality and metabolic changes in a validated murine model of pancreatic cancer cachexia. PLoS One 10: e0132786, 2015.

18. Mikami S, Mizuno R, Kosaka T, Saya H, Oya M and Okada Y: Expression of TNF- $\alpha$ and CD44 is implicated in poor prognosis, cancer cell invasion, metastasis and resistance to the sunitinib treatment in clear cell renal cell carcinomas. Int J Cancer 136: 1504-1514, 2015

19. Nguyen CB, Kumar S, Zucknick M, Kristensen VN, Gjerstad J, Nilsen $\mathrm{H}$ and Wyller VB: Associations between clinical symptoms, plasma norepinephrine and deregulated immune gene networks in subgroups of adolescent with chronic fatigue syndrome. Brain Behav Immun 76: 82-96, 2019.

20. Strawbridge R, Sartor ML, Scott F and Cleare AJ: Inflammatory proteins are altered in chronic fatigue syndrome-A systematic review and meta-analysis. Neurosci Biobehav Rev 107: 69-83, 2019.

21. Wang Q, Tu H, Zhu M, Liang D, Ye Y, Chang DW, Long Y and Wu X: Circulating obesity-driven biomarkers are associated with risk of clear cell renal cell carcinoma: A two-stage, case-control study. Carcinogenesis 40: 1191-1197, 2019.

22. Sun L, Gao Z, Luo L, Tan H and Zhang G: Estrogen affects cell growth and IGF-1 receptor expression in renal cell carcinoma. Onco Targets Ther 11: 5873-5878, 2018.

23. Kohno K, Okamoto I, Sano O, Arai N, Iwaki K, Ikeda M and Kurimoto M: Royal jelly inhibits the production of proinflammatory cytokines by activated macrophages. Biosci Biotechnol Biochem 68: 138-145, 2004.

24. Sugiyama T, Takahashi K, Tokoro S, Gotou T, Neri P and Mori H: Inhibitory effect of 10-hydroxy-trans-2-decenoic acid on LPS-induced IL- 6 production via reducing I $\kappa \mathrm{B}-\zeta$ expression. Innate Immun 18: 429-437, 2012.

25. Zargar HR, Hemmati AA, Ghafourian M, Arzi A, Rezaie A and Javad-Moosavi SA: Long-term treatment with royal jelly improves bleomycin-induced pulmonary fibrosis in rats. Can J Physiol Pharmacol 95: 23-31, 2017.

26. Eisenhauer EA, Therasse P, Bogaerts J, Schwartz LH, Sargent D, Ford R, Dancey J, Arbuck S, Gwyther S, Mooney M, et al: New response evaluation criteria in solid tumours: Revised RECIST guideline (version 1.1). Eur J Cancer 45: 228-247, 2009.

27. Miyata Y, Matsuo T, Sagara Y, Ohba K, Ohyama K and Sakai H: A mini-review of reactive oxygen species in urological cancer: Correlation with NADPH oxidases, angiogenesis, and apoptosis. Int J Mol Sci 18: 2214, 2017.

28. Takahashi K, Sugiyama T, Tokoro S, Neri P and Mori $\mathrm{H}$ Inhibition of interferon- $\gamma$-induced nitric oxide production by 10 -hydroxy-trans-2-decenoic acid through inhibition of interferon regulatory factor- 8 induction. Cell Immunol 273 $73-78,2012$.
29. Abu-Serie MM and Habashy NH: Two purified proteins from royal jelly with in vitro dual anti-hepatic damage potency: Major royal jelly protein 2 and its novel isoform X1. Int J Biol Macromol 128: 782-795, 2019.

30. Sun KH, Sun GH, Wu YC, Ko BJ, Hsu HT and Wu ST: TNF- $\alpha$ augments CXCR2 and CXCR3 to promote progression of renal cell carcinoma. J Cell Mol Med 20: 2020-2028, 2016.

31. Shin SY, Kim CG, Jung YJ, Jung Y, Jung H, Im J, Lim Y and Lee YH: Euphorbia humifusa willd exerts inhibition of breast cancer cell invasion and metastasis through inhibition of TNF $\alpha$-induced MMP-9 expression. BMC Complement Altern Med 16: 413, 2016.

32. Deng Y, Long L, Wang K, Zhou J, Zeng L, He L and Gong Q: Icariside, II, a broad-spectrum anti-cancer agent, reverses beta-amyloid-induced cognitive impairment through reducing inflammation and apoptosis in rats. Front Pharmacol 8: 39, 2017.

33. Harrison ML, Obermueller E, Maisey NR, Hoare S, Edmonds K, Li NF, Chao D, Hall K, Lee C, Timotheadou E, et al: Tumor necrosis factor alpha as a new target for renal cell carcinoma: Two sequential phase II trials of infliximab at standard and high dose. J Clin Oncol 25: 4542-4549, 2007.

34. Kawashima A, Uemura M, Kato T, Ujike T, Nagahara A, Fujita K, Imamura R, Yamanaka Y, Tomiyama E, Tanigawa G, et al: Results of weekday-on and weekend-off administration schedule of sunitinib therapy for advanced renal cell carcinoma. Int J Clin Oncol 24: 78-86, 2019.

35. Iwamoto $\mathrm{K}$, Ishihara $\mathrm{H}$, Takagi $\mathrm{T}$, Kondo $\mathrm{T}$, Yoshida $\mathrm{K}$, Iizuka $\mathrm{J}$ and Tanabe $\mathrm{K}$ : Evaluation of relative dose intensity during the early phase of first-line sunitinib treatment using a 2-week-on/1-week-off regimen for metastatic renal cell carcinoma. Med Oncol 35: 78, 2018.

36. Ohba K, Miyata Y, Yasuda T, Asai A, Mitsunari K, Matsuo T, Mochizuki Y, Matsunaga N and Sakai H: Efficacy and safety of sunitinib alternate day regimen in patients with metastatic renal cell carcinoma in Japan: Comparison with standard 4/2 schedule. Asia Pac J Clin Oncol 14: 153-158, 2018.

37. Lee L, Ito T, Igarashi H, Miki M, Fujimori N, Kawabe K, Jensen RT and Ogawa Y: Dose and schedule modification are required for long-term continuation of sunitinib in Japanese patients with advanced pancreatic neuroendocrine tumors. Cancer Chemother Pharmacol 81: 163-169, 2018.

38. Zugmaier G, Paik S, Wilding G, Knabbe C, Bano M, Lupu R, Deschauer B, Simpson S, Dickson RB and Lippman M: Transforming growth factor beta 1 induces cachexia and systemic fibrosis without an antitumor effect in nude mice. Cancer Res 51: 3590-3594, 1991.

This work is licensed under a Creative Commons Attribution-NonCommercial-NoDerivatives 4.0 International (CC BY-NC-ND 4.0) License. 